\title{
Sistema de Informação Geográfica no mapeamento de arboviroses no Território de Desenvolvimento Entre Rios, Estado do Piauí, Brasil
}

\section{Geographic Information System in the mapping of arboviruses in the Entre Rios Development Territory, State of Piauí, Brazil}

DOI: $10.46919 / \operatorname{archv} 1 n 3-004$

Recebimento dos originais: 10/03/2020

Aceitação para publicação: 10/04/2020

\author{
Romário Gonçalves da Silva \\ Graduado em Geografia pela Universidade Federal do Piauí - UFPI \\ Instituição: Universidade Federal do Piauí - UFPI \\ Endereço: Campus Ministro Petrônio Portela - UFPI - Ininga, Teresina -PI, Brasil \\ E-mail: romariogs38@gmail.com \\ Vívian Ribeiro Magalhães \\ Graduanda em Geografia pela Universidade Federal do Piauí \\ Instituição: Universidade Federal do Piauí - UFPI \\ Endereço: Campus Ministro Petrônio Portela - UFPI - Ininga, Teresina -PI, Brasil \\ E-mail: vivianrm1401@hotmail.com \\ Antonio Carlos dos Santos \\ Doutorando em Desenvolvimento e Meio Ambiente pela rede PRODEMA/UFPI \\ Instituição: Universidade Federal do Piauí - UFPI \\ Endereço: Av. Universitária, 1310 (TROPEN) 64.049-550 Teresina - Piauí - Brasil \\ E-mail: baulive@hotmail.com
}

\section{Emanuel Lindemberg Silva Albuquerque}

Doutor em Geografia pela Universidade Estadual do Ceará - UECE

Instituição: Universidade Federal do Piauí - UFPI

Endereço: Campus Ministro Petrônio Portela - UFPI - Ininga, Teresina -PI, Brasil.

E-mail: lindemberg@ufpi.edu.br

\section{RESUMO}

O espaço geográfico tem sido objeto de estudo em diversas perspectivas, sendo que a Geografia da Saúde vem ganhando destaque em razão do crescente aumento de doenças que vêm sendo notificadas na esfera global. Nesse sentido, esse processo tem potencializado, por sua vez, novas ferramentas geoinformacionais que auxiliam no estudo, na análise e no mapeamento das doenças ao nível espacial. Diante do exposto, essa pesquisa objetiva realizar o mapeamento de arboviroses no Território de Desenvolvimento Entre Rios, no Estado do Piauí, considerando o recorte temporal de 2012 a 2016, na perspectiva de modelar em ambiente de Sistema de Informação Geográfica (SIG), os setores territoriais que apresentam as maiores incidências no número de casos de dengue. Para tanto, foi necessário estruturar um SIG por meio de um inventário digital, com capacidade de interligar as informações em um banco de dados, permitindo a realização de consultas e a apresentação dos dados na forma de mapas temáticos. Deste modo, foi possível traçar o perfil situacional dos municípios do Território de Desenvolvimento Entre Rios, frente à ocorrência dos arbovírus da dengue. A pesquisa em epígrafe visou 
o desencadeamento de ações mais efetivas ao nível territorial, a partir do viés de transformar os dados em informação e, consequentemente, em conhecimento.

Palavras-chave: Geoprocessamento, Arboviroses, Dengue.

\begin{abstract}
The geographic space has been the object of study in several perspectives, and the Geography of Health has been gaining prominence due to the growing increase of diseases that have been reported in the global sphere. In this sense, this process has, in turn, potentialized new geoinformational tools that assist in the study, analysis and mapping of diseases at the spatial level. Given the above, this research aims to map arboviruses in the Entre Rios Development Territory, in the State of Piauí, considering the time frame from 2012 to 2016, in the perspective of modeling in a Geographic Information System (GIS) environment, the sectors areas that have the greatest impact on the number of dengue cases. For this, it was necessary to structure a GIS through a digital inventory, with the ability to link information in a database, allowing queries and data to be presented in the form of thematic maps. In this way, it was possible to trace the situational profile of the municipalities in the Entre Rios Development Territory, in view of the occurrence of dengue arboviruses. The research in question aimed to trigger more effective actions at the territorial level, from the perspective of transforming data into information and, consequently, into knowledge.
\end{abstract}

Keywords: Geoprocessing, Arboviroses, Dengue.

\title{
1 INTRODUÇÃO
}

Em virtude de ser o estudo da ocorrência de arboviroses uma temática fundamental na percepção integrada de doenças, torna-se necessário mencionar que o estudo em epígrafe visa destacar, do ponto de vista territorial, a ocorrência dos casos de dengue no Território de Desenvolvimento Entre Rios, no Estado do Piauí, a partir do mapeamento geoespacial.

Menezes (2001) menciona que a quantificação de indicadores na saúde permite comparações na população, sendo que estes podem refletir, com fidedignidade, o panorama da saúde num determinado território. O autor menciona que é interessante observar que esses indicadores apesar de serem denominados indicadores de saúde, muitos deles medem doenças, o que denota ser mais fácil, às vezes, medir doença do que medir saúde, como é o caso da espacialização da ocorrência de arboviroses, a exemplo da dengue.

Vale salientar que a dengue é uma doença febril viral, do grupo das arboviroses, transmitida pelo díptero Aedes aegypti, que provoca sérios problemas em várias regiões do mundo. Menciona-se que metade da população mundial vive sob o risco da doença, que teve um expressivo registro de crescimento nas últimas décadas (WHO, 2014), sendo que sua forma hemorrágica pode levar a óbito (GUBLER, 2002).

Menezes (2001) menciona que a quantificação de indicadores na saúde permite comparações na população, sendo que estes podem refletir, com fidedignidade, o panorama da saúde num determinado 
território. Não obstante, vale ressaltar que a dengue é a mais importante arbovirose que afeta o homem. No Brasil as arboviroses (dengue, zika e chicungunya) tem causado muito problemas de saúde pública na última década (LOPES et al., 2018; VALLADARES et al., 2019).

No Piauí, a presença do Aedes aegypti foi confirmada primeiramente no ano de 1986. Em 1994, levantamentos entomológicos realizados pela Fundação Nacional de Saúde (Funasa), confirmaram os primeiros casos autóctones de dengue. No ano de 1998 foi constatada uma epidemia no Estado, sendo registrados 10.081 casos e 4 óbitos com maior incidência da doença no primeiro semestre (MONTEIRO et al., 2009, PIAUÍ, 2018).

Ao considerar o exposto, esse trabalho objetiva realizar o mapeamento de arboviroses no Território de Desenvolvimento Entre Rios, no Estado do Piauí, considerando o recorte temporal de 2012 a 2016, na perspectiva de modelar em ambiente de Sistema de Informação Geográfica (SIG), os setores territoriais que apresentam as maiores incidências no número de casos de dengue.

Deste modo, foi possível traçar, com base nos 31 municípios do mencionado Território de Desenvolvimento, evidências temporais e espaciais relacionadas aos casos de incidência do vírus da dengue, na perspectiva de prever tendências de epidemia ao nível territorial, pois o dado/informação/conhecimento possibilita uma melhor tomada de decisão por parte do poder público e da sociedade civil organizada.

\section{MATERIAL E MÉTODOS}

No presente artigo além do estudo geográfico ambiental da espacialidade, que possibilita o entendimento da dinâmica da organização espacial (CAVALCANTI, 2006), foi utilizado também o epidemiológico, com abordagem retroativa, do tipo ecológico e ambiental, por se referir à área geográfica estudada (base municipal), com variáveis ambientais, habitacionais e indicadores de saúde (ALMEIDA FILHO; ROUQUAYROL,1990; ALMEIDA; MEDRONHO; VALENÇA, 2009).

A pesquisa (qualitativa-quantitativa) encontra-se estruturada por meio do levantamento de dados bibliográficos de autores como: Carvalho, Pina e Santos. (2000), Câmara e Monteiro (2001), Menezes (2001), Câmara et al. (2004), Monteiro et al. (2009) e entre outros, obtidos por meio de livros, artigos científicos e sites especializados sobre o assunto.

Dessa forma, Câmara et al. (2004) destaca que para compreender a distribuição espacial de dados oriundos de fenômenos ocorridos no espaço em ambiente SIG, constitui hoje um grande desafio para a elucidação de questões centrais, tendo em vista as complexidades que são permeadas no espaço geográfico, a exemplo da coleta de dados primários.

Portanto, a coleta dos dados epidemiológicos dos casos de dengue da presente pesquisa foi obtida por meio das informações do Sistema de Informação de Agravos de Notificação (SINAN), 


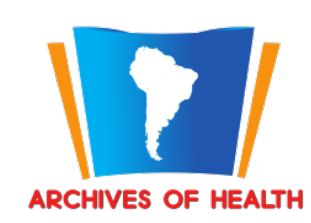

integrantes da base de dados da Secretaria de Estado da Saúde do Piauí (SESAPI), sendo que os limites municipais das 31 cidades integrantes do Território de Desenvolvimento Entre Rios, no Estado do Piauí, foram adquiridos no site do Instituto Brasileiro de Geografia e Estatística (IBGE).

Na sequência, em ambiente SIG, foi possível fazer a junção de dados vetoriais e alfanuméricos, através da função union do ArcGis. Em seguida, na opção (Open Attribute table) e em (Add field) foi gerado uma nova coluna para calcular o total de casos de dengue no período de 2012 à 2016, no intuito de produzir o mapa com a distribuição espacial dos casos notificados na área em destaque, o que possibilitou a geração do mapa gráfico em forma de pizza, através da opção (Symbology-Charts-Pie).

Vale salientar que todo o trabalho foi desenvolvido no Laboratório de Geografia Física e Estudos Ambientais - GEOAMBIENTE, da Coordenação do Curso de Geografia (CGEO/CCHL), da Universidade Federal do Piauí (UFPI), fruto do projeto de iniciação científica, e utilizando-se do software de geoprocessamento ArcGis 10.5 (licença estudantil) que culminou com o mapeamento geoespacial do Território de Desenvolvimento Entre Rios, utilizando-se do Sistema de Projeção Geográfica, tendo como referencial geodésico o Sistema de Referência Geocêntrico para as Américas (SIRGAS 2000).

\section{RESULTADOS E DISCUSSÕES}

De posse da base de dados estruturada e georreferenciada ao nível municipal, foi possível proceder a espacialização dos resultados ao nível do Território de Desenvolvimento Entre Rios, sendo que os dados denotam a heterogeneidade do vetor nos municípios e no recorte temporal em destaque (Figura 1). É importante frisar que o mapa apresenta os casos notificados de dengue ao nível municipal, contudo, devido a dinamicidade do espaço geográfico, não se deve levarem consideração para a análise do vetor apenas os limites dos municípios, mas sobretudo os fatores que contribuem para a ocorrência e proliferação deste, considerando o quantitativo notificado nos anos de 2012 a 2016 (Tabela 1). 


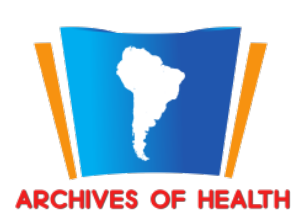

Figura 1 - Distribuição anual dos casos de dengue no Território de Desenvolvimento Entre Rios, Estado do Piauí

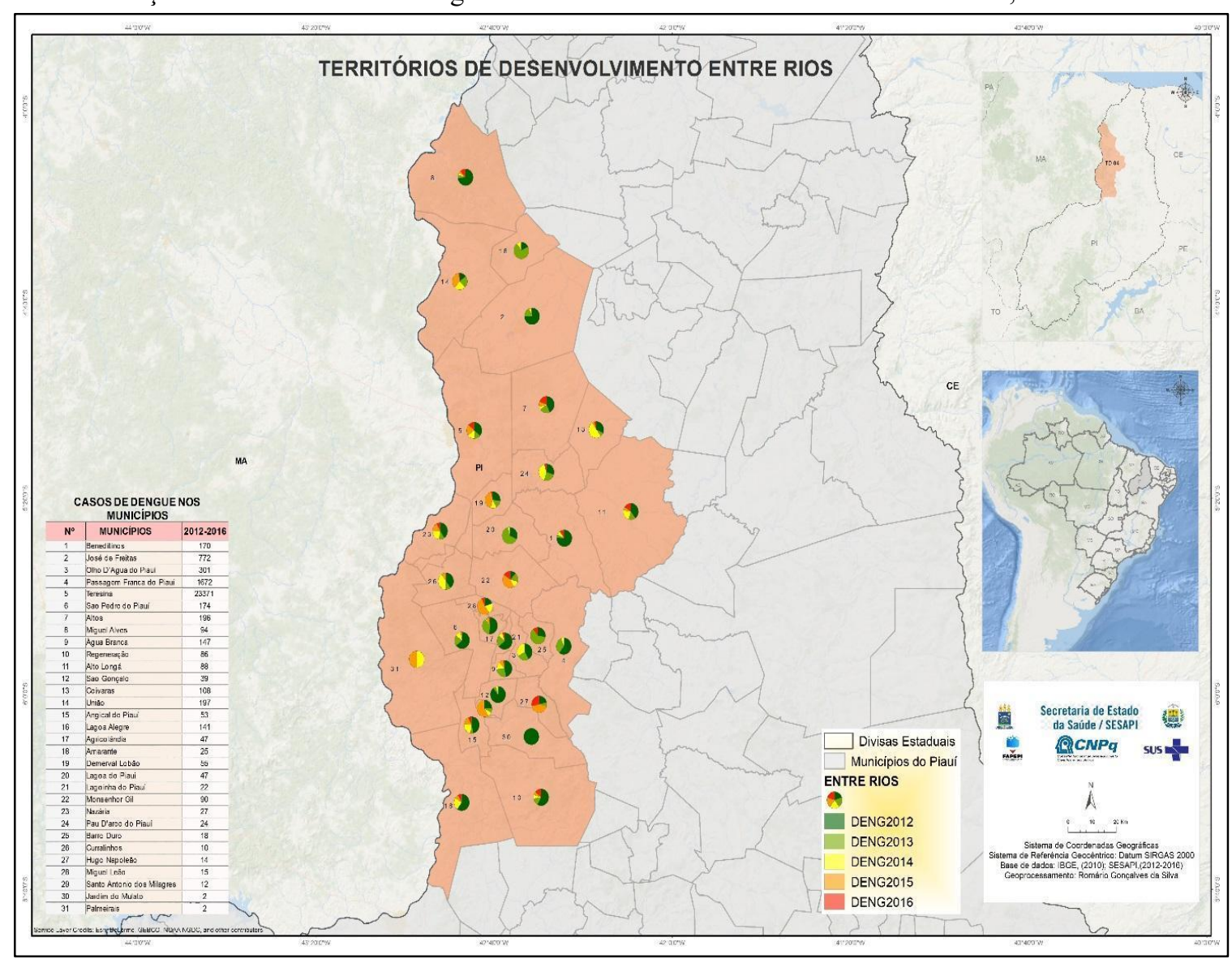

Fonte: IBGE (2010); SESAPI (2012-2016). Elaboração: Autores (2018).

Tabela 1 - Número de casos notificados de dengue entre os anos de 2012-2016 no Território de Desenvolvimento Entre Rios, Estado do Piauí

\begin{tabular}{c|c}
\hline Período & Número de casos totais notificados no TD entre rios \\
\hline 2012 & 11081 \\
\hline 2013 & 3965 \\
\hline 2014 & 4370 \\
\hline 2015 & 5297 \\
\hline 2016 & 3306 \\
\hline
\end{tabular}

Fonte: Secretaria do Estado da Saúde do Piauí (2012-2016).

Com base nos dados analisados, o município de Teresina, segundo dados do IBGE (2010) é o município do Piauí que apresenta a maior população, são 861.442 habitantes, sendo que destes, 94\% são residentes na zona urbana e 16\% na zona rural, apresentando densidade demográfica de 584,94 hab/ $\mathrm{km}^{2}$ e índice de esgotamento saneamento de 61,6 \%, sendo este o município com o maior número de casos notificados (23.371), o que representa um grande problema não só pela sua importância enquanto capital, mas devido às ocorrências de novas doenças veiculadas pelo mesmo agente transmissor (Aedes Aegypti).

Por outro lado, o município de Passagem Franca do Piauí, ao sul do Território de Desenvolvimento Entre Rios, também apresentou expressivo número de casos de dengue, sendo que 
foram registradas 1.672 notificações, porém diferente de Teresina, o município apresenta apenas 4.546 habitantes, densidade demográfica da ordem de 5,35 hab $/ \mathrm{km}^{2}$ e esgotamento saneamento em torno de 8,9\%. Na sequência, aparece o município de José de Freitas, que apresentou no recorte temporal 772 casos, sendo que sua população é de $37.085 \mathrm{hab} / \mathrm{km}^{2}$, e o esgotamento sanitário corresponde a 39\%.

Menciona-se que o Território de Desenvolvimento Entre Rios, do ponto de vista geográfico, encontra-se dentro da zona intertropical, área propícia para a proliferação de doenças veiculadas por artrópodes da família culicidae, sendo que a proximidade com a Linha do Equador apresenta um maior aumento da evaporação, devido às elevadas temperaturas e por apresentar chuvas em alguns meses do ano, habituando a população a armazenar água em cisternas, caixa d'água, manilhas e outros, propiciando o local ideal para que o mosquito venha a estabelecer seus ovos e, por conseguinte, sua reprodução.

Portanto, não se deve analisar a doença apenas por um único fator, pois mesmo com uma alta densidade demográfica não significa que a ocorrência de surto, endemia ou epidemia da doença possa ocorrer nesse local, pois com a dispersão do vetor pode haver uma epidemia em área que não apresente casos da doença.

\section{CONCLUSÃO}

Em virtude do exposto, a dispersão espaço-temporal dos vetores ocorre devido à variação das condições ambientais e antrópicas das cidades, o que favorece a dinâmica entre o vetor-homem-espaço, pois essa interação propicia a proliferação do Aedes aegypti e aumento de casos em diferentes escalas de adversidade do agente etiológico.

Nesse sentido, a dispersão do vetor no Território de Desenvolvimento Entre Rios contribuiu para um total de 28.019 casos notificados, resultado dos processos climáticos, acrescidos do crescimento urbano e a forte influência antrópica, variáveis que tendem a contribuir para a dispersão do vetor, conforme constatado entre os anos de 2012 a 2016.

Portanto, a espacialização dos dados, por meio do mapa gerado, tende a subsidiar, com conhecimento mais preciso, as ações das secretarias de saúde do Estado e dos municípios, pois se torna imprescindível, em termos de controle do vetor da doença, o planejamento e controle das ações quanto ao progresso e/ou retrocesso dos casos confirmados de dengue no Território de Desenvolvimento Entre Rios, no Estado do Piauí. 


\section{AGRADECIMENTOS}

Os autores agradecem ao Programa Pesquisa para o SUS: gestão compartilhada em saúde (PPSUS), no âmbito do Edital FAPEPI/MS-DECIT/CNPq/SESAPI $\mathrm{N}^{\circ} 002 / 2016$, pelo financiamento do projeto intitulado Avaliação geoespacial da ocorrência de arboviroses no estado do Piauí: um enfoque na dengue e no zika vírus, bem como a Gerência de Vigilância em Saúde da Secretaria de Estado da Saúde do Piauí (SESAPI), pela parceria de trabalho.

\section{REFERÊNCIAS}

ALMEIDA, A. S., MEDRONHO, R. A., VALENCIA, L. I. O. Análise espacial da dengue e o contexto socioeconômico no município do Rio de Janeiro, RJ. Revista de Saúde Pública, v. 43, p. 666-673, 2009.

ALMEIDA FILHO, N.; ROUQUAYROL, M. Z. Introdução a Epidemiologia Moderna. Coedição ABRASCO. Apce Produtos do Conhecimento. 1990.

CÂMARA, G.; MONTEIRO, A. M.; FUCKS, S. D.; CARVALHO, M. S.. Analise espacial e geoprocessamento. In: "Análise Espacial de Dados Geográficos". Brasília, EMBRAPA, 2004. p. 136. Disponível em:< http://www.dpi.inpe.br/gilberto/livro/analise/>. Acesso em: 7 ago. 2018.

CAVAlCANTI, A. P. B. Métodos e Técnicas da Pesquisa Ambiental. UFPI/CCHL/DGH, Teresina PI, 2006.

CARVAlHO, M. S.; PINA, M. F.; SANTOS, S. Conceitos básicos de Sistemas de Informação Geográfica e Cartografia aplicados à saúde. Brasília: OPAS, 2000.

CÂMARA, G.; MONTEIRO, A. M.;. Conceitos básicos em ciência da geoinformação. In: Introdução à Ciência da Geoinformação. CÂMARA, Gilberto; DAVIS, Clodoveu; MONTEIRO, Antônio Miguel Vieira (org.) São José dos Campos, INPE, 2001. p.2-35.

GUBLER, D.J. Epidemic dengue/dengue hemorrhagic fever as a public health, social and economic problemin the 21st century. Trends in microbiology, v. 10, n. 2, p. 100-103, 2002.

LOPES, T. R. R.; SILVA, C. S.; PASTOR, A. F.; SILVA JÚNIOR, J. V. J. Dengue in Brazil in 2017: whathappened?.Revista do Instituto de Medicina Tropical de São Paulo, v. 60, 2018. https://doi.org/10.1590/s1678-9946201860043

MENEZES, A. M. B. Noções básicas de epidemiologia. In: MENEZES, A. M. B. Epidemiologia das doenças respiratórias. Rio de Janeiro: Revinter, 2001. p. 1-25.

MONTEIRO, E. S. C.; COELHO, M. E.; CUNHA, I. S.; CAVAlCANTE, M. A. S.; CARVALHO, F. A. A. Aspectos epidemiológicos e vetoriais da dengue na cidade de Teresina, Piauí - Brasil, 2002 a 2006. Epidemiol. Serv. Saúde, Brasília, n. 18, v4, p. 365-374, out-dez, 2009. 
PIAUÍ. SECRETARIA DE ESTADO DA SAÚDE DO PIAUÍ - SESAPI. Dengue, Chikungunya, Zika e Microcefalia. Boletim da 14 ${ }^{\text {a }}$ Semana Epidemiológica. Teresina: Secretaria de Estado da Saúde do Piauí. 5 p. 2018.

VALLADARES, GUSTAVO SOUZA ; HASSUM, Izabella Cabral ; ALBUQUERQUE, EMANUEL LINDEMBERG SILVA; SANTOS, ANTÔNIO CARLOS DOS . INFLUÊNCIA DE VARIÁVEIS AMBIENTAIS NA OCORRENCIA DA DENGUE UTILIZANDO GEOPROCESSAMENTO EM TERESINA, PIAUÍ. Hygeia.Revista Brasileira de Geografia Médica e da Saúde, v. 15, p. 102-114, 2019.

WORLD HEALTH ORGANIZATION. Dengue and severe dengue. WHO, 2014. 